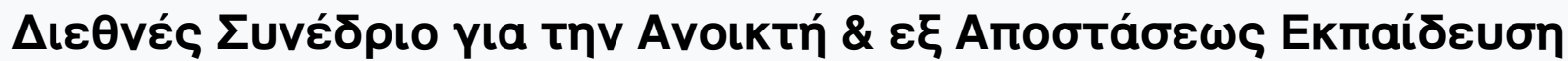

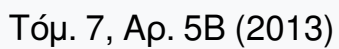

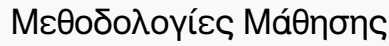

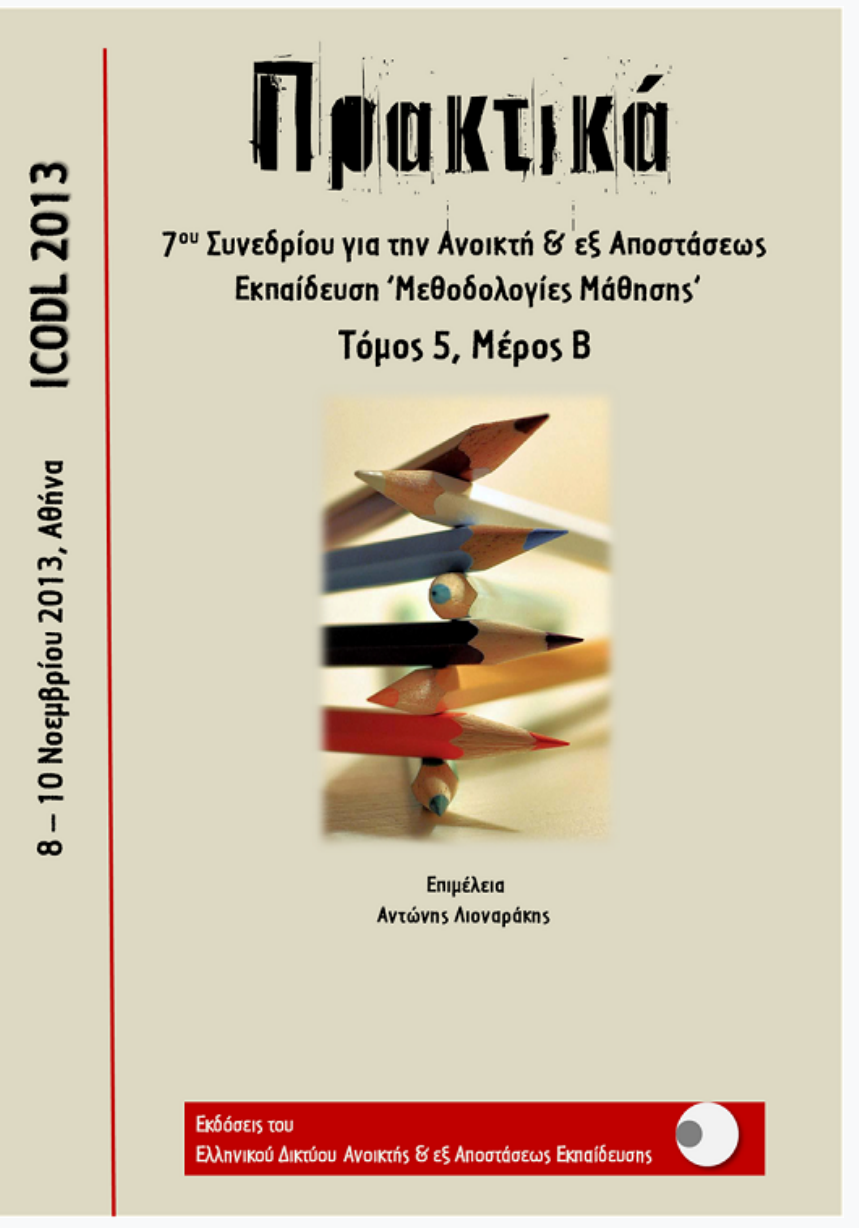

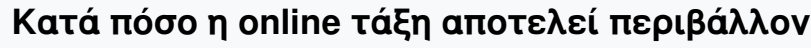

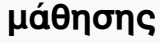

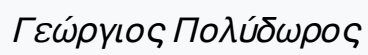

doi: $\underline{10.12681 / \text { icodl.549 }}$ 


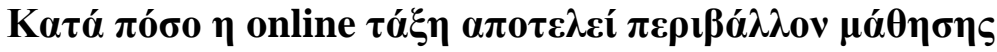

\section{Is online class a learning environment?}

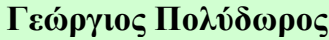

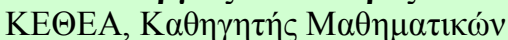 \\ Yлочท́ \\ georgiospolydoros@gmail.com
}

\begin{abstract}
This paper is a literature review which examines whether online education has the capacity to support effective learning. The effectiveness of learning should be the first criteria to judge any delivery method. Thus, if the students in online education cannot learn as well as in traditional classes, then all other critical online issues, including access, student's and teacher's satisfaction, etc., have no meaning. The following are presented, based on the studied literature: The research paper focuses in the technological tools that the methodology uses and presents the online's distance education researchers' findings on the learning efficacy. Furthermore, it examines the methodology's characteristics and its relationship with the learning theories. Finally, in conclusion, the research shows that the online methodology's learning quality and the learning outcomes are just about the same compared to face-face learning outcomes. Findings are showing that the delivery method is just as effective as traditional education but online education is not surpassing traditional education, yet. The role of online education has changed from an alternative learning to a protagonist in most universities. In spite of the numerous studies in this area no definitive answer to the question "are the learning results in online classes similar compared to those of traditional education?" has been found.
\end{abstract}

Keywords: online class, online education, learning, effectiveness

\section{Пєрі́ $\eta \psi \eta$}

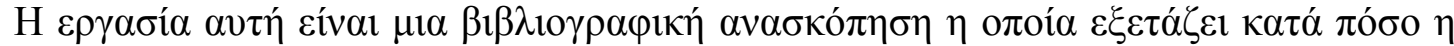

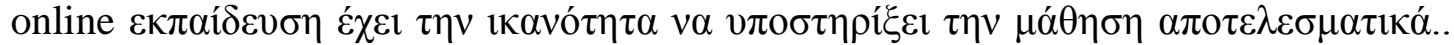

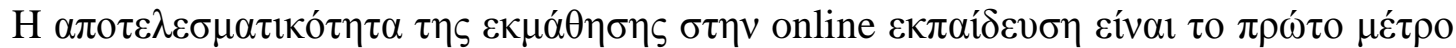

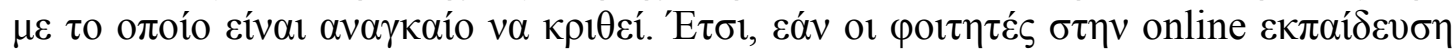

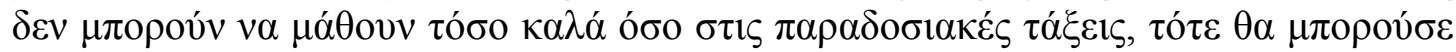

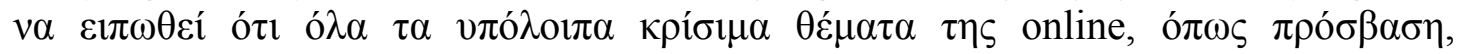

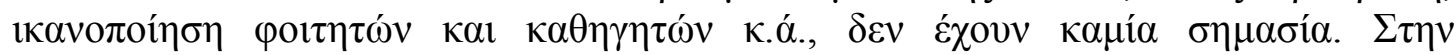

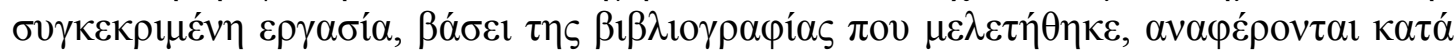

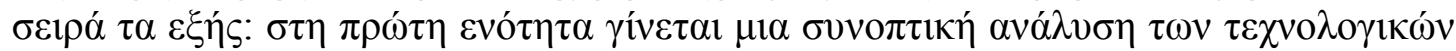

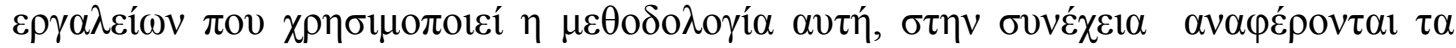

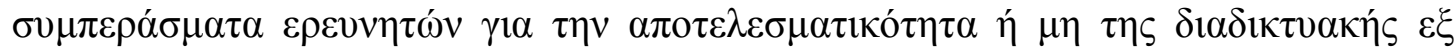

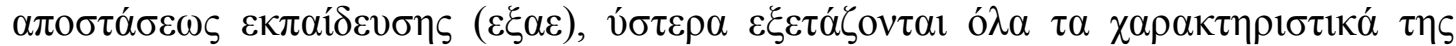

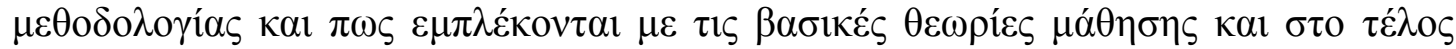

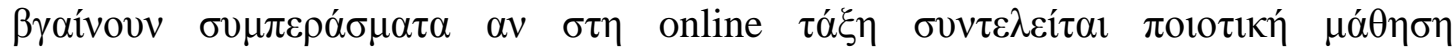

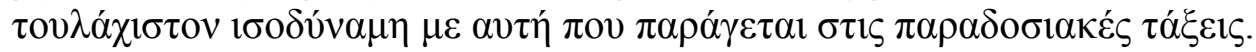




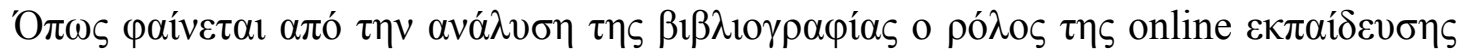

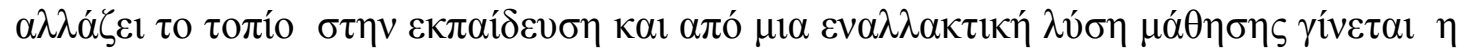

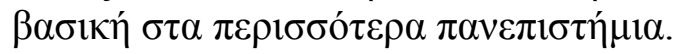

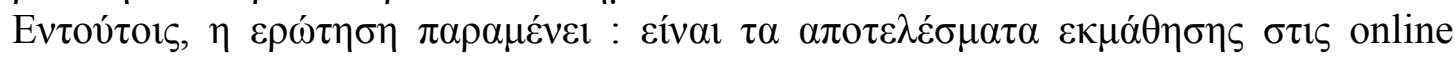

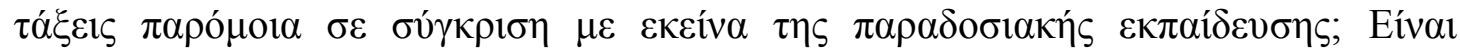

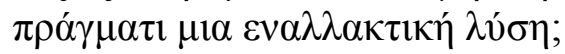

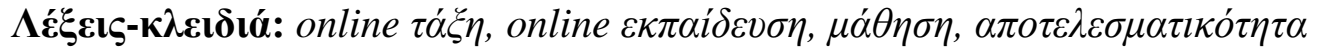

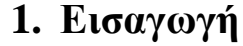

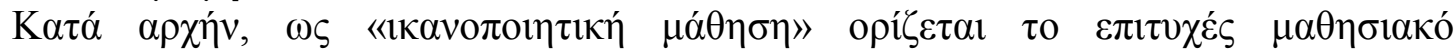

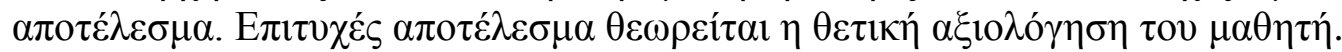

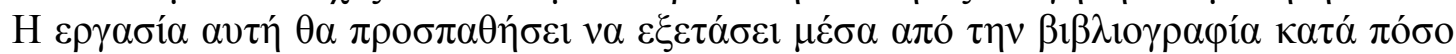

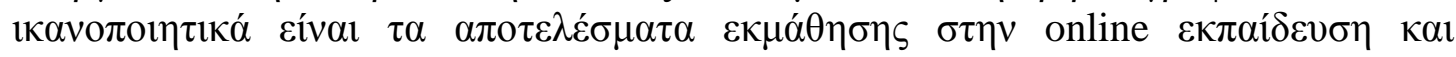

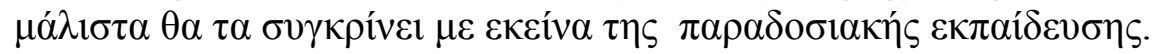

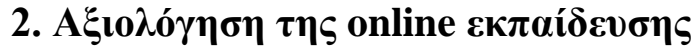

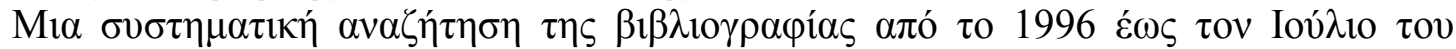

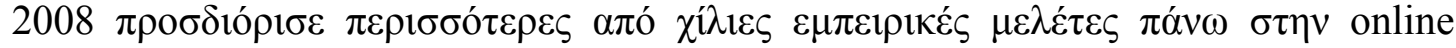

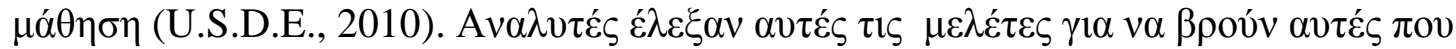
(U.S.D.E., 2010): $\alpha$ ) $\alpha v \tau \iota \pi \alpha \rho \alpha \beta \alpha ́ \lambda \lambda$ ovv $\tau \eta \nu$ online $\mu \varepsilon \tau \eta v \pi \rho o ́ \sigma \omega \pi \mathrm{o} \mu \varepsilon \pi \rho o ́ \sigma \omega \pi \mathrm{o}$

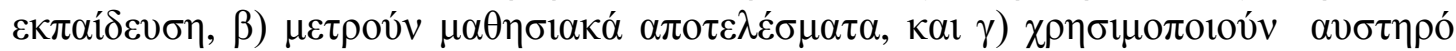

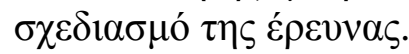

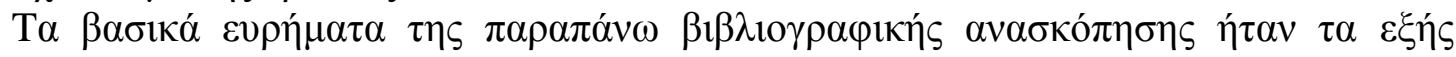
(U.S.D.E., 2010):

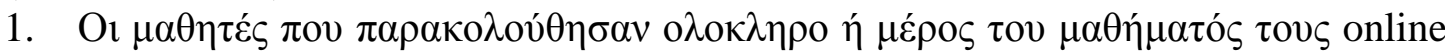

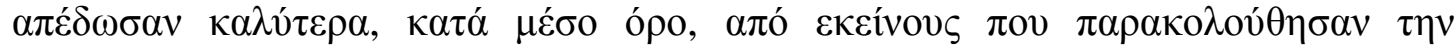
$\pi \alpha \rho \alpha \delta$ обакท́ $\delta 1 \delta \alpha \sigma \kappa \alpha \lambda i ́ \alpha \pi \rho o ́ \sigma \omega \pi \mathrm{o} \mu \varepsilon \pi \rho \sigma_{\sigma} \sigma \omega \pi \mathrm{o}$.

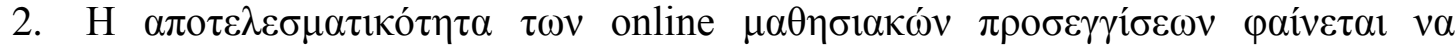

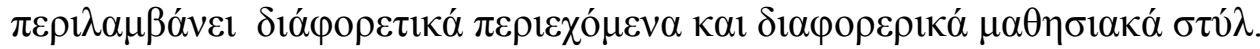

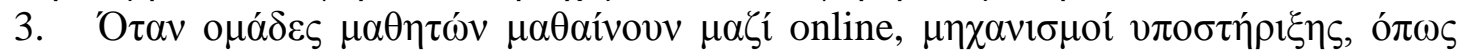

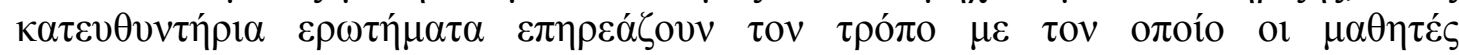
$\alpha \lambda \lambda \eta \lambda \varepsilon \pi 1 \delta \rho \circ v^{2}$.

\section{Online $\varepsilon \kappa \pi \alpha i ́ \delta \varepsilon v \sigma \eta$}

\subsection{H online $\mu \varepsilon \theta 0 \delta o \lambda o \gamma i ́ a$}

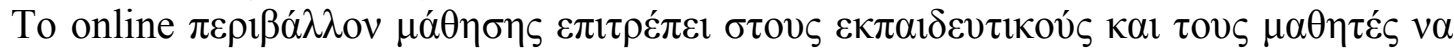

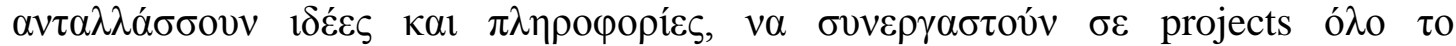

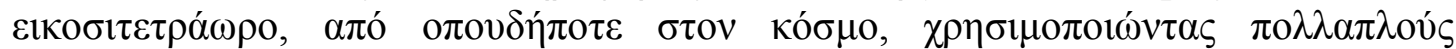

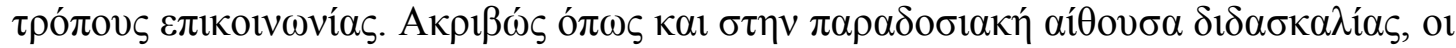

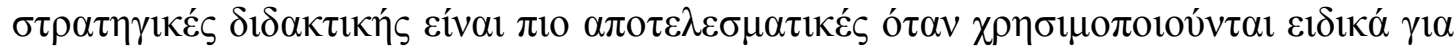

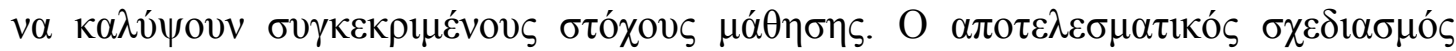

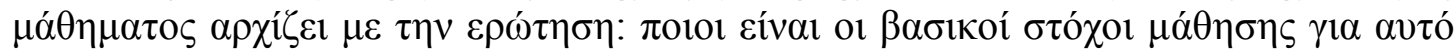

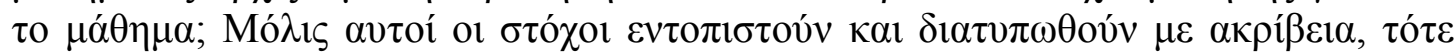

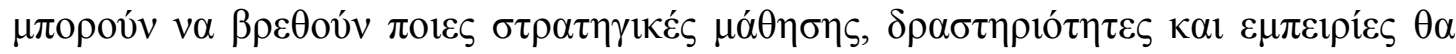

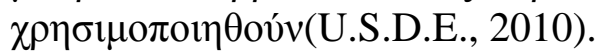

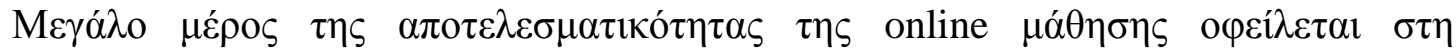

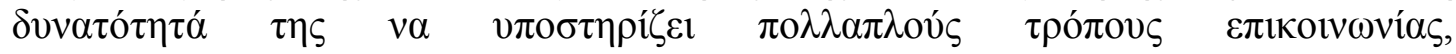

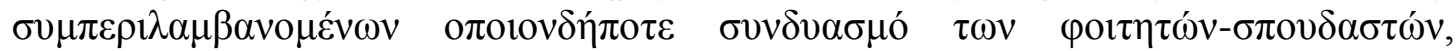




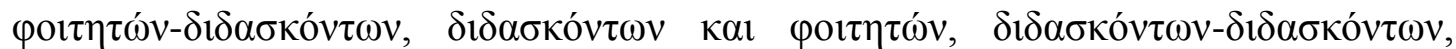

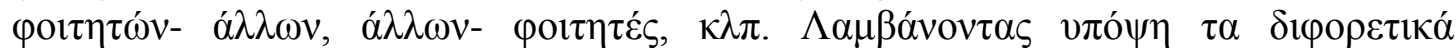

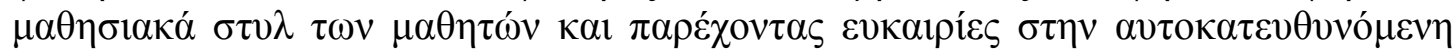

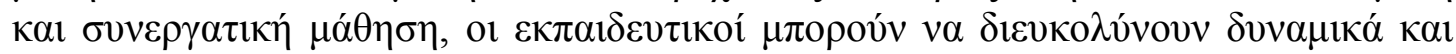

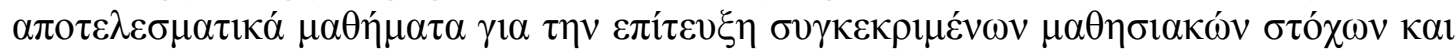

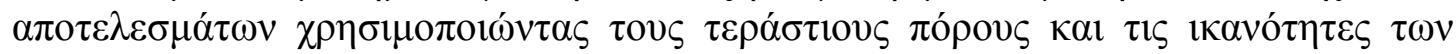

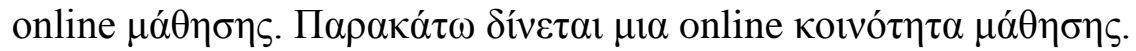

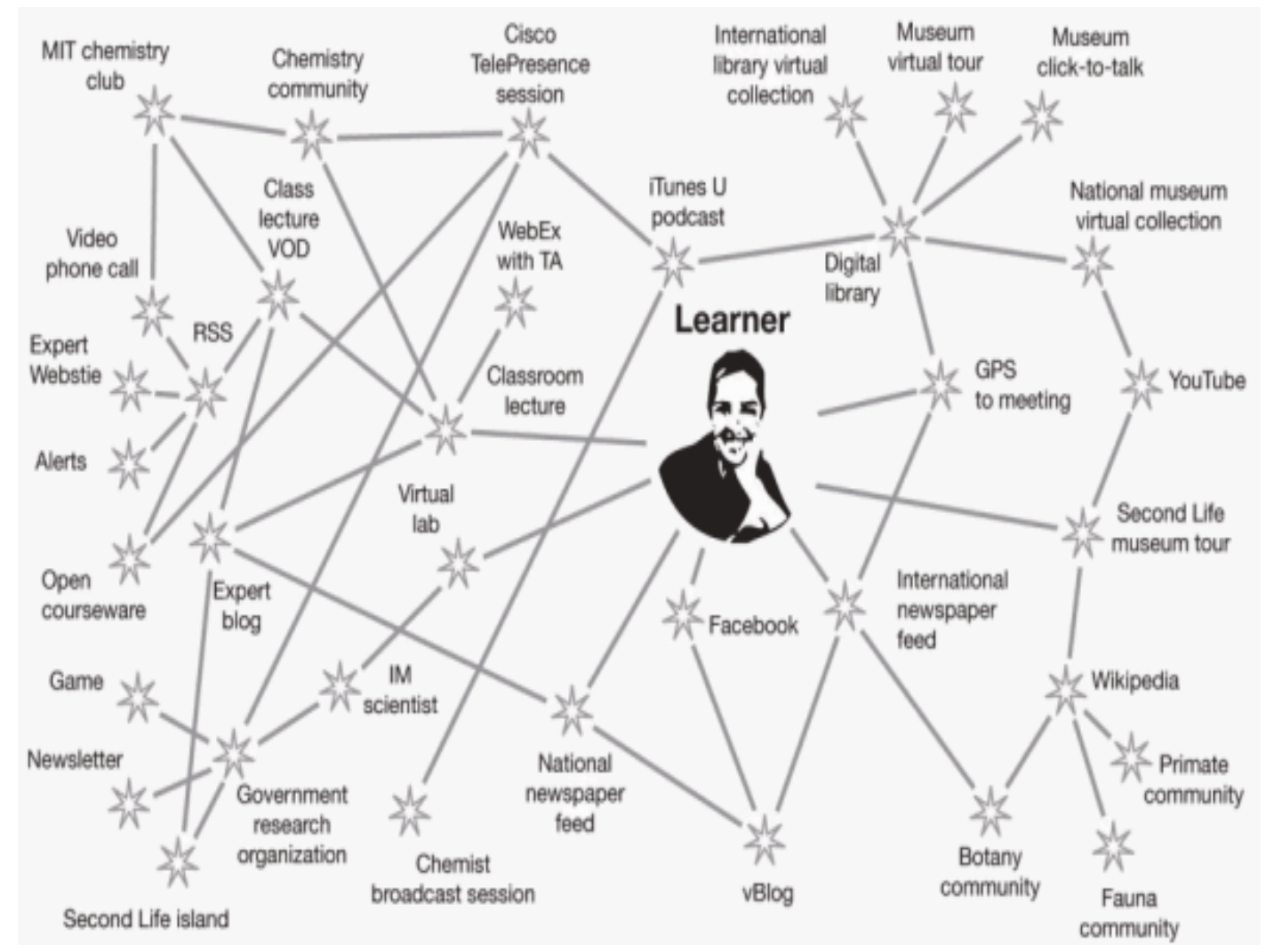

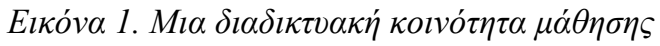

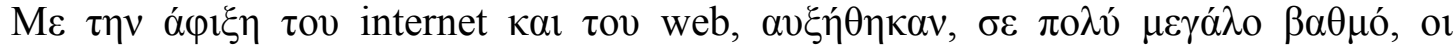

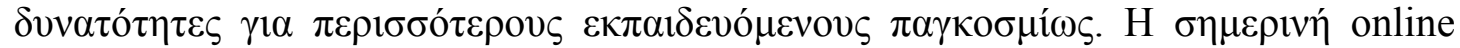

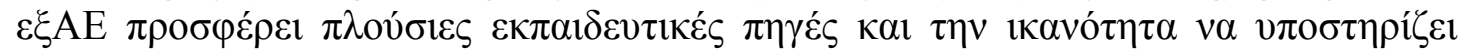

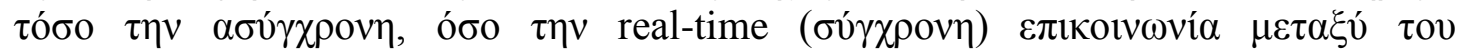

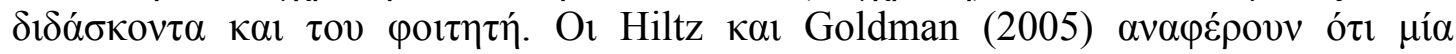

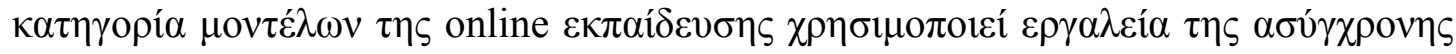
$\varepsilon \pi ı \kappa o v \omega v i ́ \alpha \zeta(\pi \cdot \chi$. email, threaded discussion boards, newsgroups), $\varepsilon v \omega ́$ á $\lambda \lambda \varepsilon \varsigma$

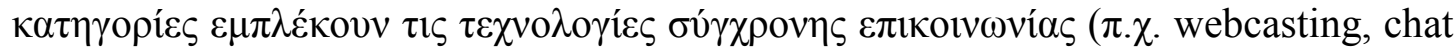

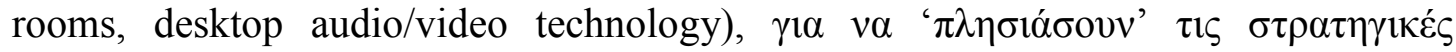

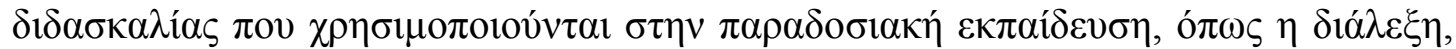

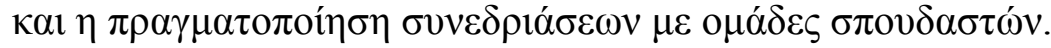

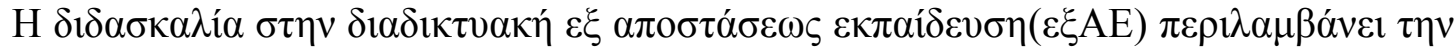

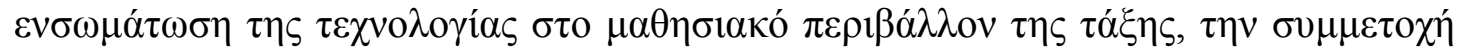

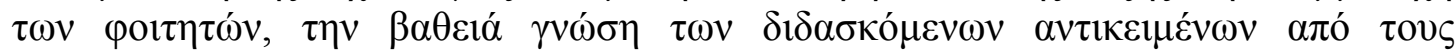

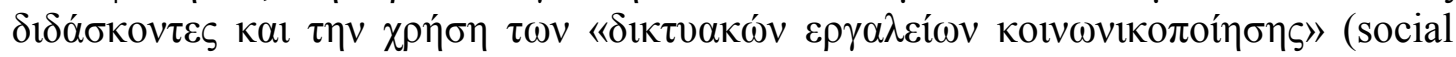
networking tools) (Larreamendy - Joerns \& Leinhardt, 2006). 


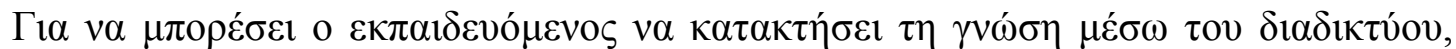

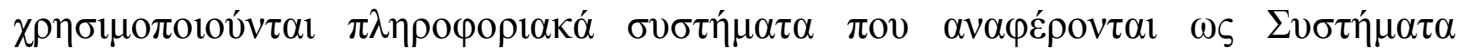

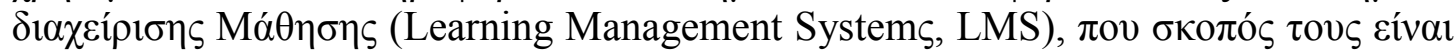

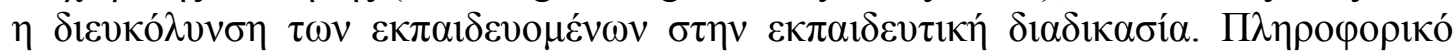

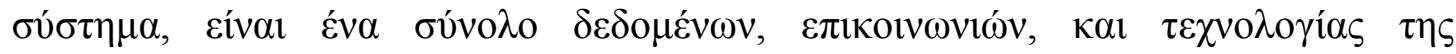

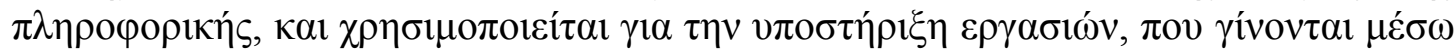

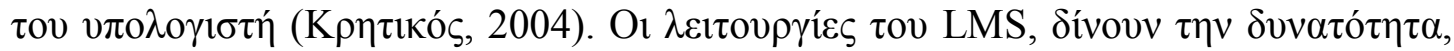

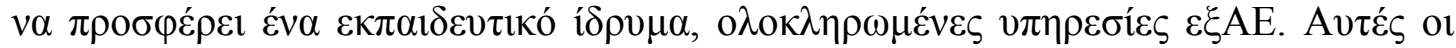

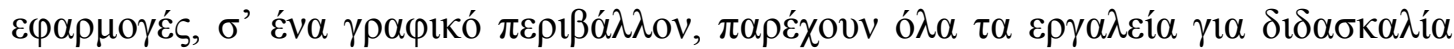

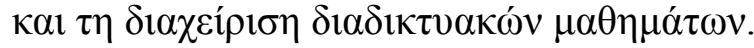

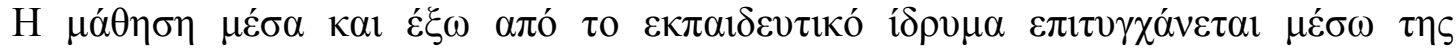

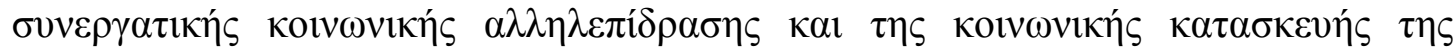

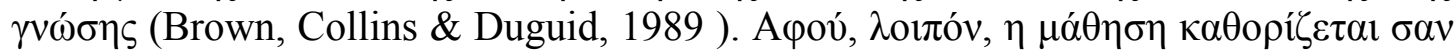

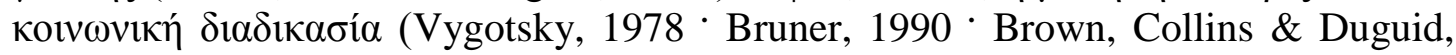

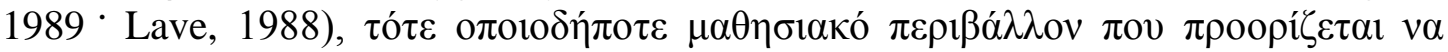

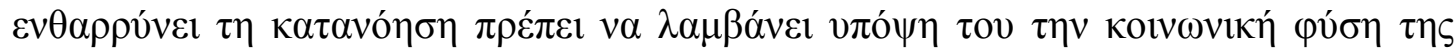

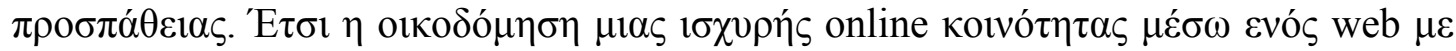

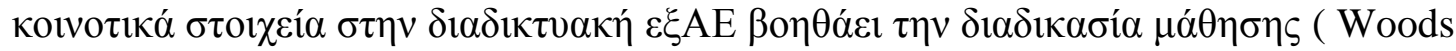
\& Ebersole, 2003).

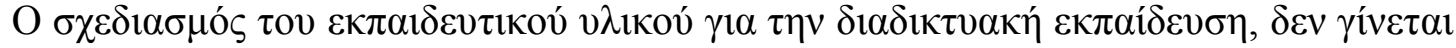

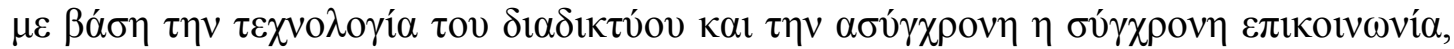

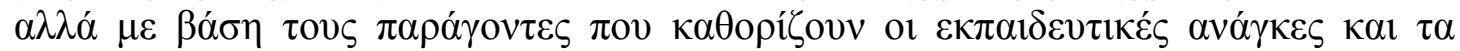

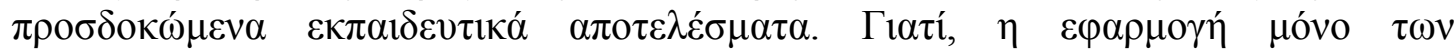

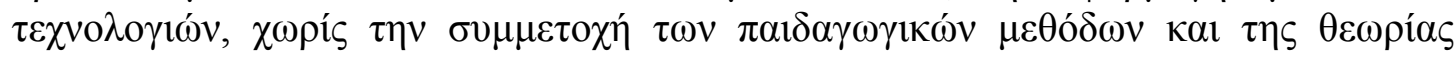

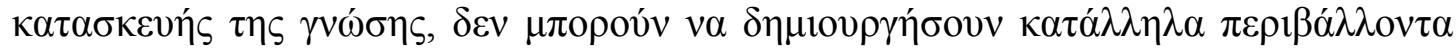
$\mu \alpha \dot{\theta} \eta \sigma \eta \varsigma$ (Kanuka, 2002).

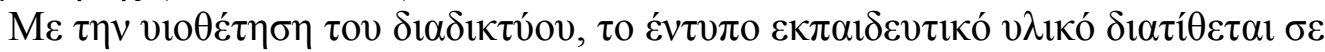

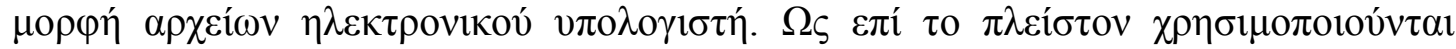

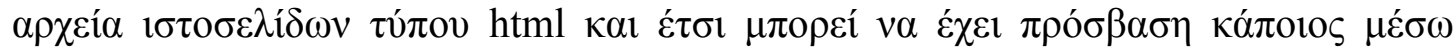

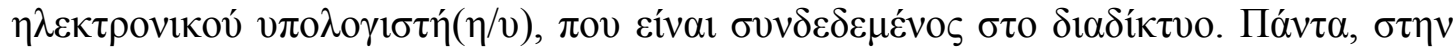

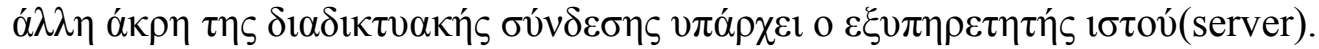

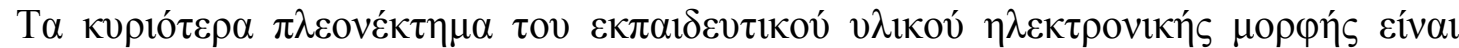
(Weller, 2000):

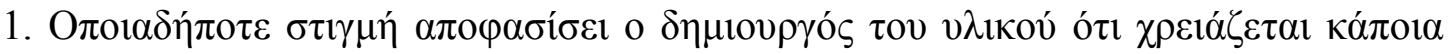

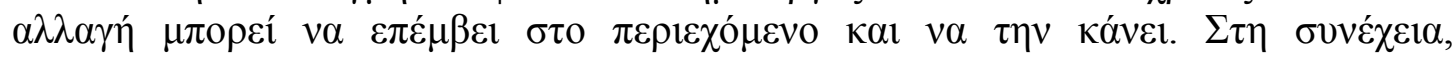

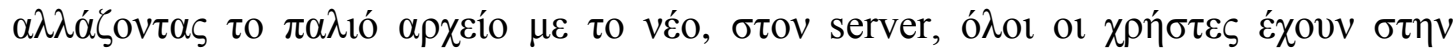

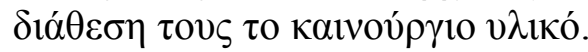

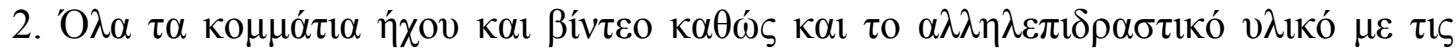

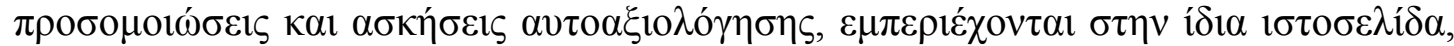

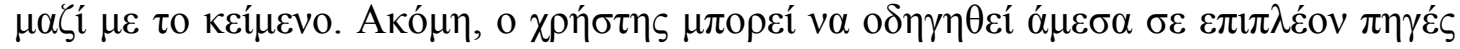

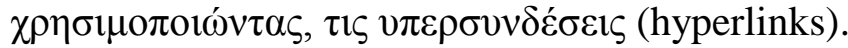

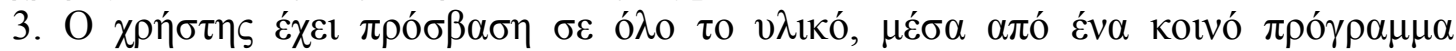

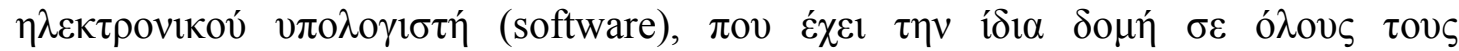

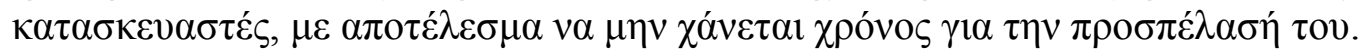

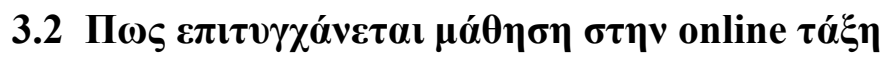

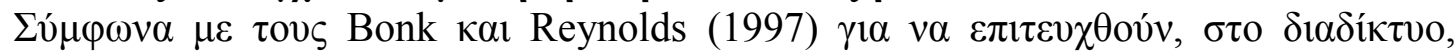

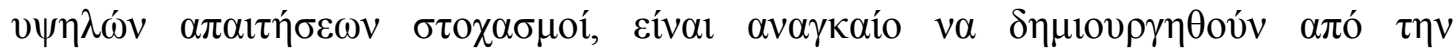

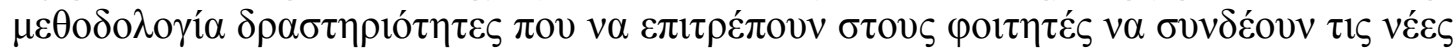




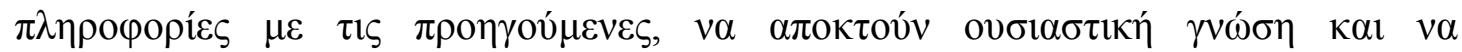

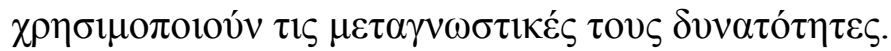

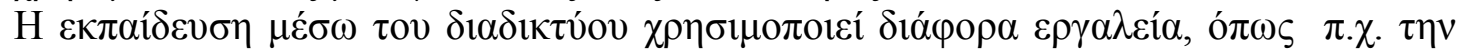

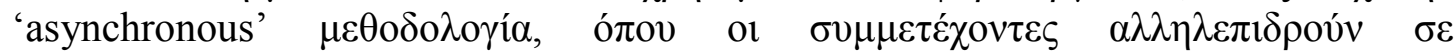

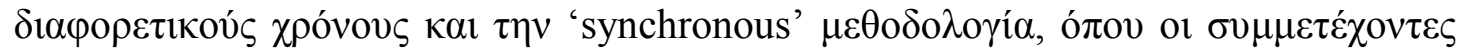

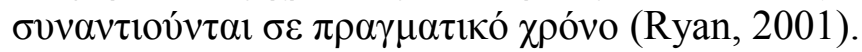

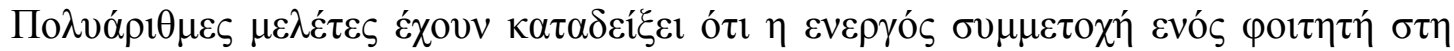

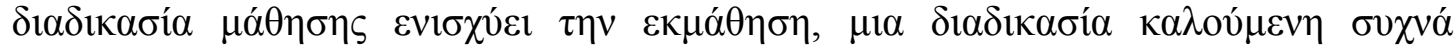

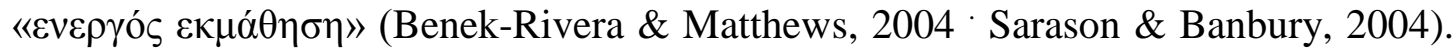

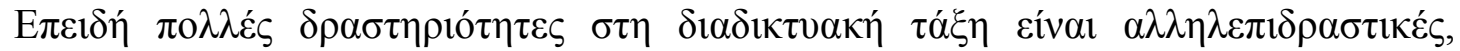

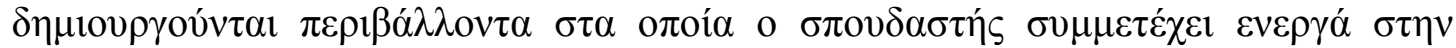

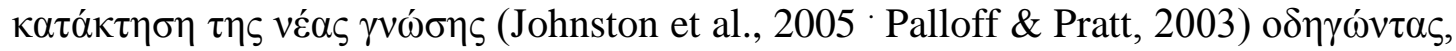

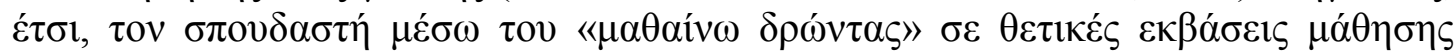
(Picciano, 2000 'Watkins, 2005).

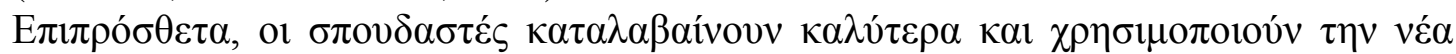

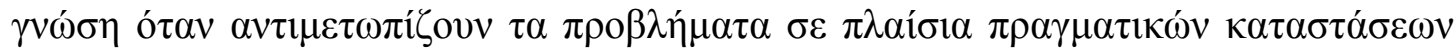

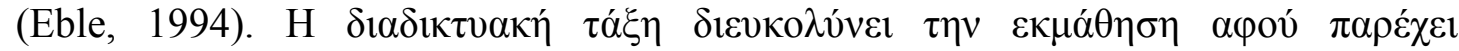

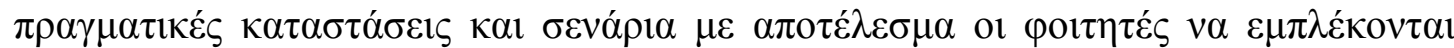

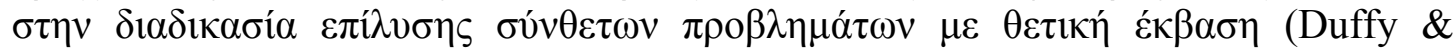

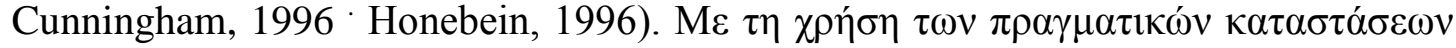

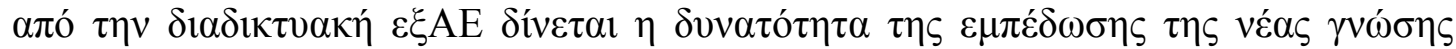

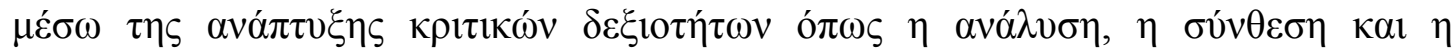

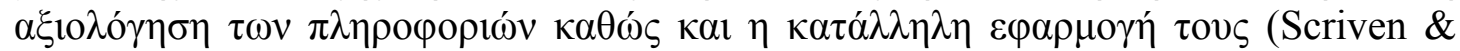

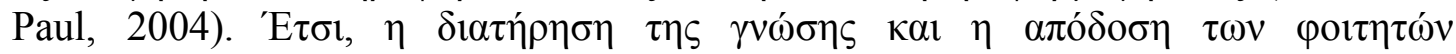

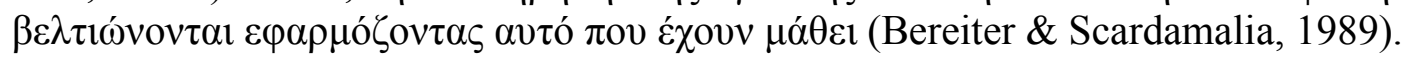

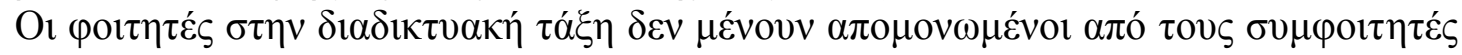

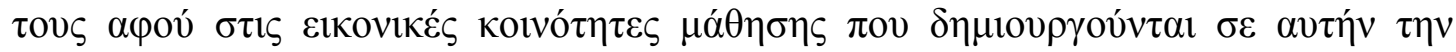

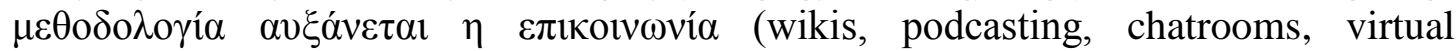

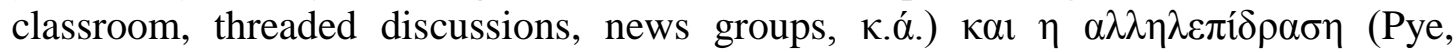

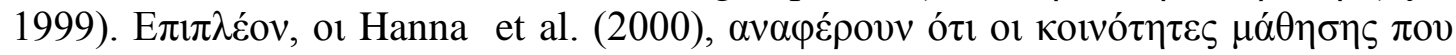

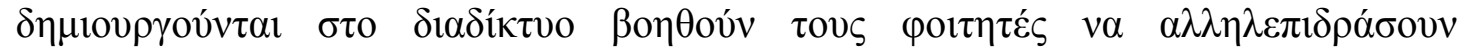

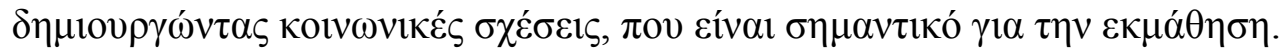

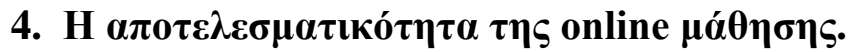

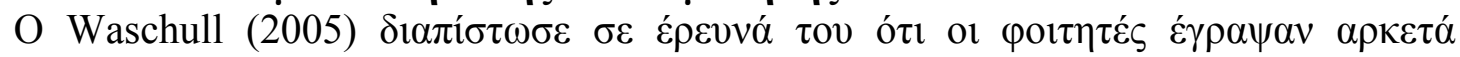

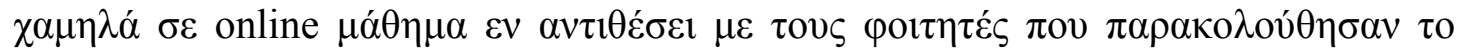

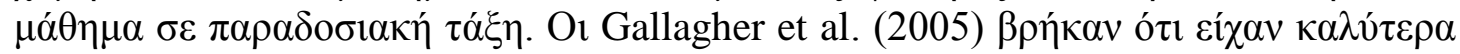

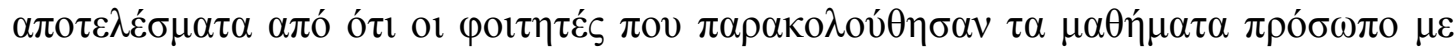

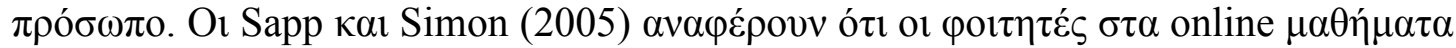

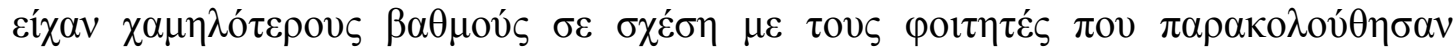

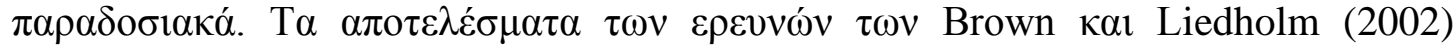

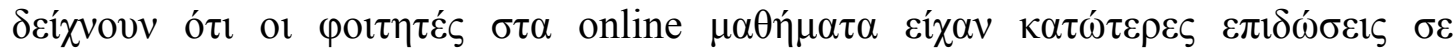

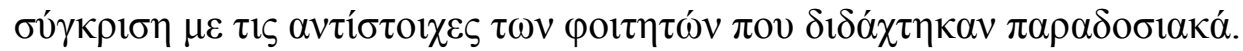

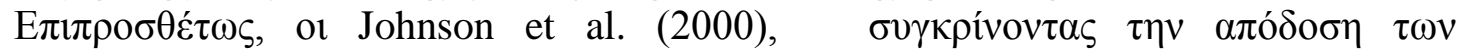

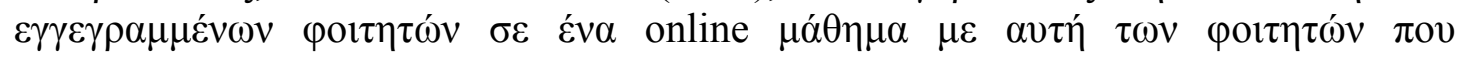

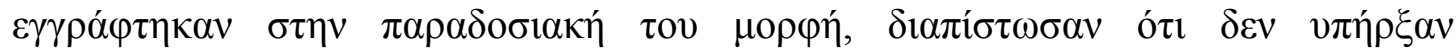

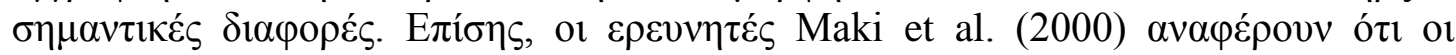

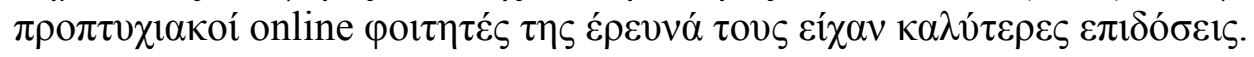

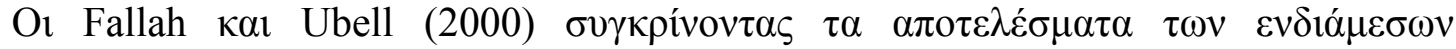

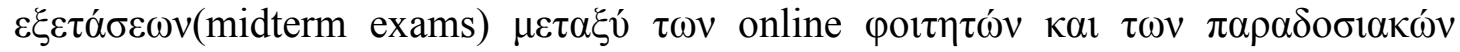




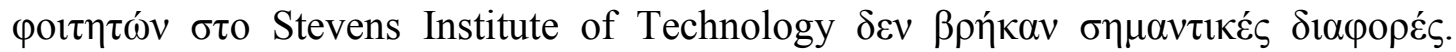

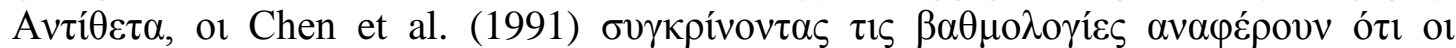

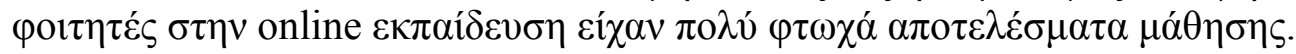

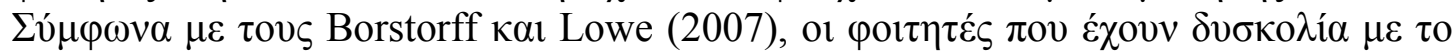

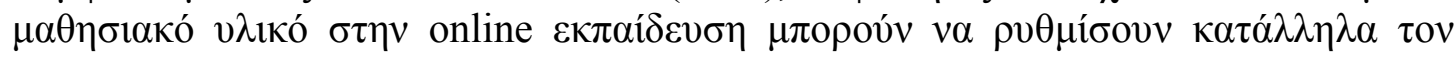

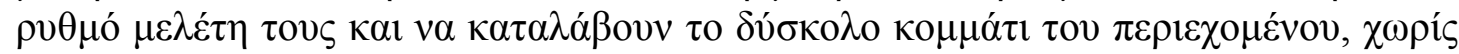

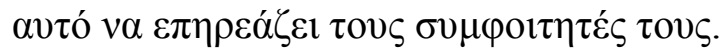

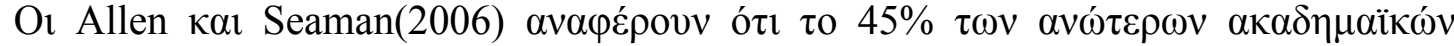

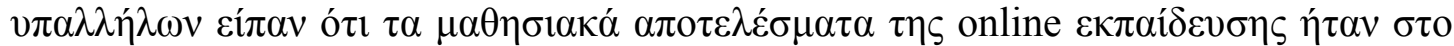

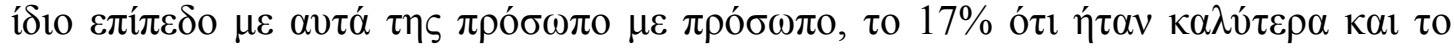

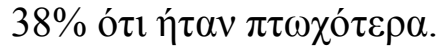

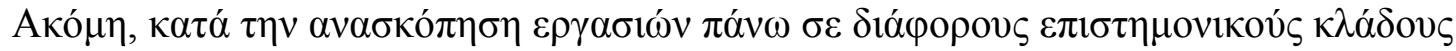

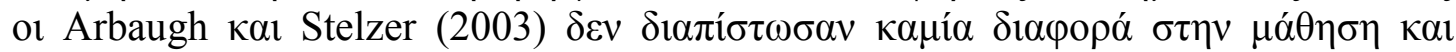

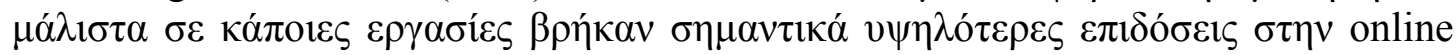

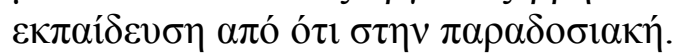

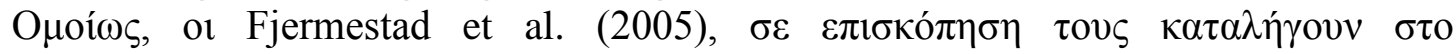

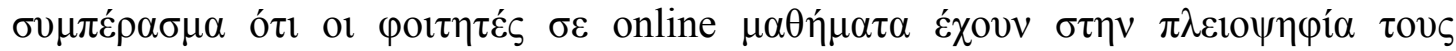

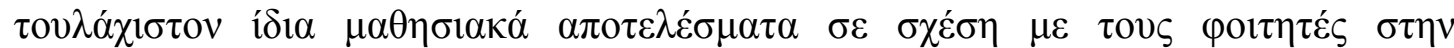

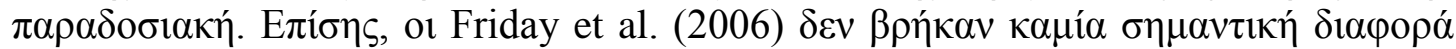

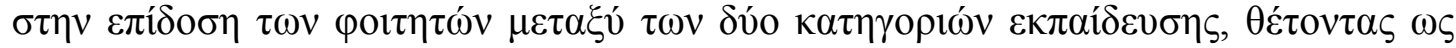

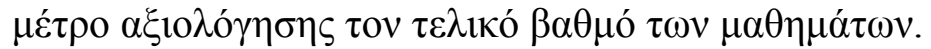

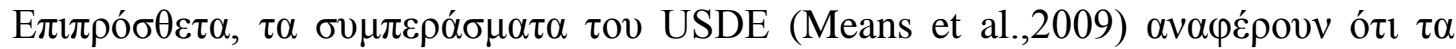

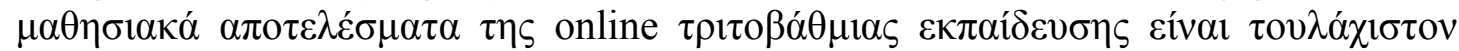

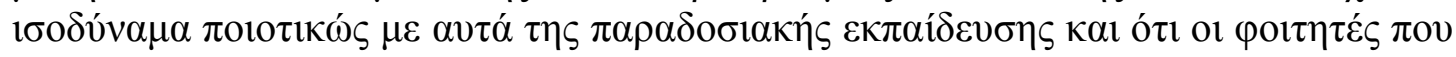

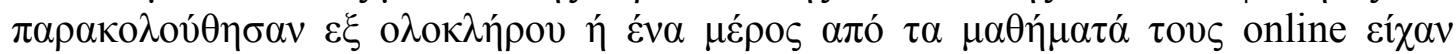

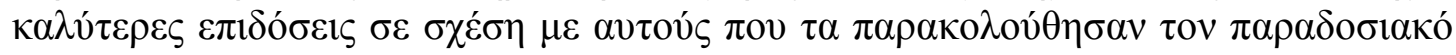

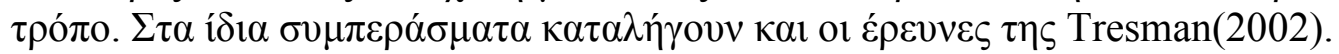

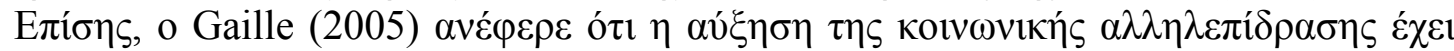

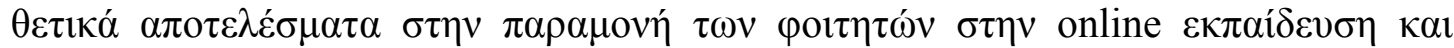

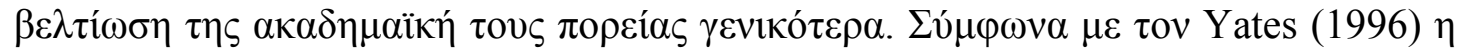

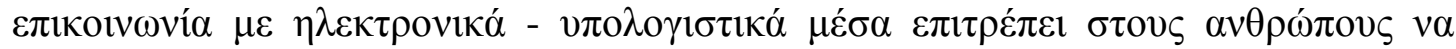

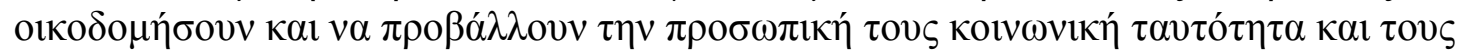

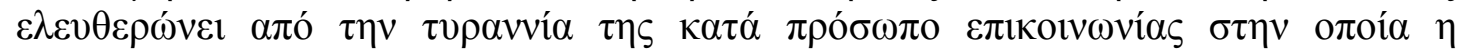

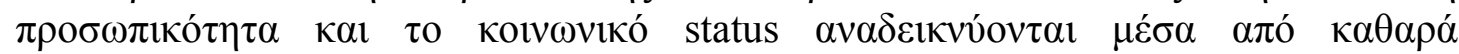

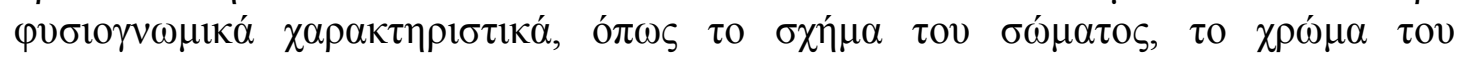

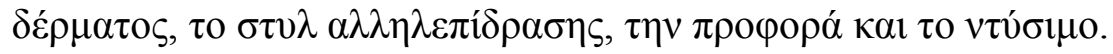

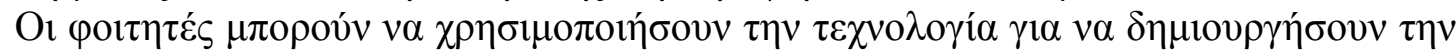

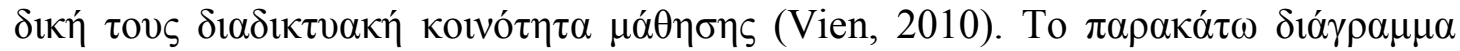

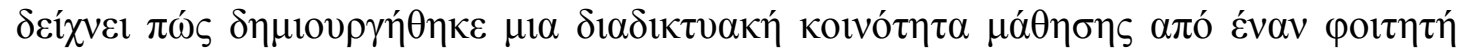

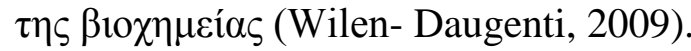

\section{6. $\Sigma v ́ v o \psi \eta-\Sigma v \mu \pi \varepsilon \rho \alpha ́ \sigma \mu \alpha \tau \alpha$}

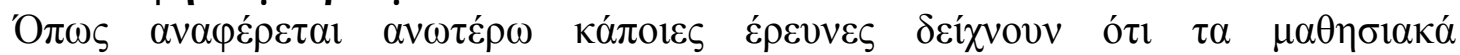

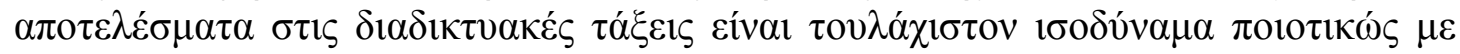

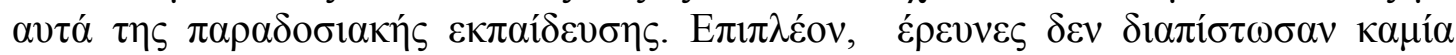

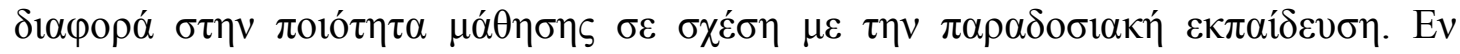

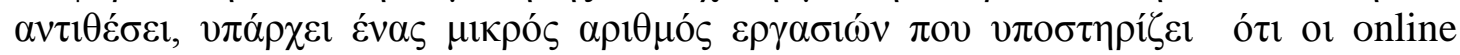

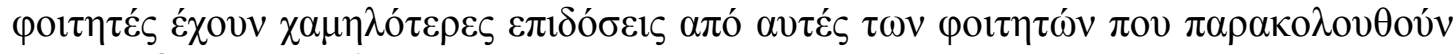

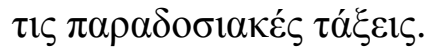




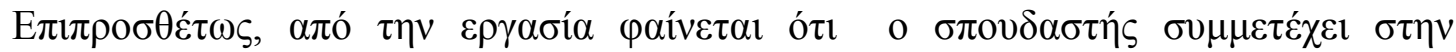

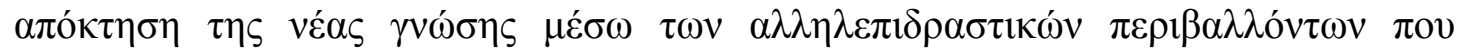

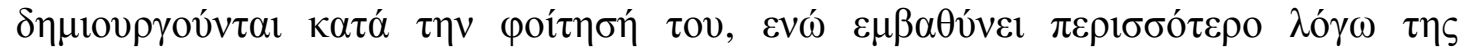

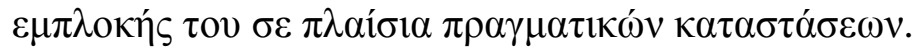

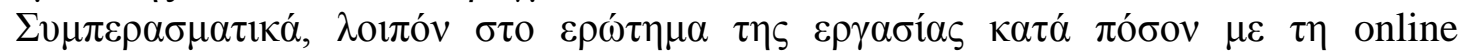

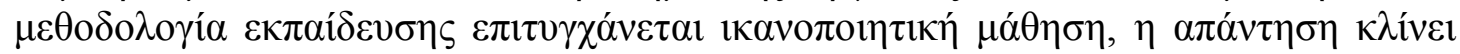

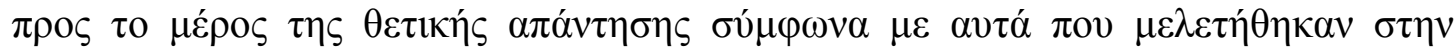
$\pi \alpha \rho \circ \sigma_{\sigma \alpha} \varepsilon \rho \gamma \alpha \sigma i ́ \alpha$.

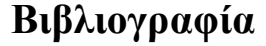

Allen, I. E. \& Seaman, J. (2006). Making the Grade: Online Education in the United States. Needham, MA: The Sloan Consortium.

Arbaugh, J. B \& Stelzer, L. (2003). Learning and teaching via the web: what do we know? $\Sigma \tau$ o C. Wankel \& R. DeFillippi (eds.) Educating Managers with Tomorrow's Technologies (pp. 1751). Greenwich, CT: Information Age Publishing.

Benek-Rivera, J. \& Matthews, V.E. (2004). Active learning with jeopardy: Students ask the questions. Journal of Management Education, 28, 104-118.

Bereiter, C. \& Scardamalia, M. (1989). Intentional learning as a goal of instruction. $\Sigma \tau$ o L.B. Resnick (Ed.), Knowing, Learning, and Instruction (p. 361-392). Hillsdale, NJ: Erlbaum.

Bonk, C.J., \& Reynolds, T.H. (1997). Learner-centered web instruction for higher-order thinking, teamwork, and apprenticeship. $\Sigma \tau$ o B. H. Khan (Ed.), Web-based instruction (p.p. 167-178). Englewood Cliffs, NJ: Educational Technology Publications.

Borstorff, P., C., Lowe, S., K. (2007). Student Perceptions and Opinions toward e-learning in the College Environment. Academy of Educational Leadership Journal, 11(2), 13-30. $\Delta 1 \alpha \theta \varepsilon \dot{\sigma} \sigma \mathrm{o}$ $\sigma \tau \eta \delta 1 \varepsilon v ́ \theta v v \sigma \eta \quad$ http://www.questia.com/library/1G1-175110710/student-perceptions-andopinions-toward-e-learning

Brown, B. \& Liedholm, C. (2002). Teaching Microeconomics: Can Web Courses Replace the Classroom in Principles of Microeconomics? The American Economic Review: Papers and Proceedings of the One Hundred and Fourteenth Annual Meeting of the American Economic Association, 79(2), 444-448.

Brown, J. S., Collins, A., \& Duguid, S. (1989). Situated cognition and the culture of learning. Educational Researcher, 18(1), 32-42.

Bruner, J. (1990). Acts of Meaning. Cambridge, MA: Harvard University Press. CAUSE/EFFECT, (1996). Campus Profile: University of Phoenix. CAUSE/EFFECT, 19(3), 28-31.

Chen, Hui-Chuan, Lehman, J., \& Armstrong, P. (1991). Comparison of performance and attitude in traditional and computer conferencing classes. American Journal of Distance Education, 5(3), $51-64$.

Duffy, T. M., \& Cunningham, D. J. (1996). Constructivism: Implications for the design and delivery of instruction. $\Sigma \tau$ o D. H. Jonassen (Ed.). Handbook of research for educational communications and technology. New York: Macmillan.

Eble, K. (1994). Craft of teaching: A guide to mastering the professor's art (2nd edition).New York: Jossey-Bass.

Fallah, M.H., \& Ubell, R. (2000). Blind scores in a graduate test: conventional compared with webbased outcomes. ALN Magazine, 4(2).

Fjermestad, J., Hiltz, S., R. \& Zhang, Y. (2005). Effectiveness for Students: comparisons of "in-seat" and ALN courses. $\Sigma \tau$ o S.R. Hiltz \& R. Goldman (Eds.) Learning Together Online: Research on Asynchronous Learning Networks (pp. 39-80). Mahwah, NJ: Lawrence Erlbaum Associates.

Friday, E., Friday-Stroud, S., Green, A., \& Hill, A. (2006). A multi- semester comparison of student performance between multiple traditional and online sections of two management courses. Journal of Behavioral and Applied Management, 8(1), 66-81.

Gaille, K. (2005). Student attrition before and after modifications in distance course delivery. Studies in Learning, Evaluation, Innovation and Development, 2(3), 69-76.

Gallagher, J., Dobrosielski-Vergona, K., Wingard, R., \& Williams, T. (2005). Web-based vs. Traditional Classroom Instruction in Gerontology: A Pilot Study. Journal of Dental Hygiene, 79(3), 1-11. 
Hanna, D. E., Glowacki-Dudka, M., \& Conceicao-Runlee, S. (2000). 147 practical tips for teaching online groups: Essentials of web-based education. Madison, WI: Atwood Publishing.

Hiltz, S. R. \& Goldman, R., Eds. (2005). Learning Together Online: Research on Asynchronous Learning Networks. Mahwah, NJ: Lawrence Erlbaum.

Honebein, P. C. (1996). Seven goals for the design of constructivist learning environments. $\Sigma \tau$ o B. G. Wilson (Ed.): Constructivist learning environments: Case studies in instructional design. Englewood Cliffs, NJ: Educational Technology Publications.

Johnston, J., Killion, J., \& Oomen, J. (2005). Student satisfaction in the virtual classroom. The Internet Journal of Allied Health Sciences and Practice.

$\Delta 1 \alpha \theta \varepsilon \dot{\sigma} \mu$ o $\quad \sigma \tau \eta \quad \delta i \varepsilon v ́ \theta v v \sigma \eta \quad$ http://ijahsp.nova.edu/articles/vol3num2/Johnston\%20$\% 20$ Printer\%20Version.pdf

Johnson, S.., Aragon, S., \& Shaik, N. (2000). Comparative analysis of learner satisfaction and learning outcomes in online and fact-to-face learning environments. Journal of Interactive Learning Research, 11 (1), 29-49.

Kanuka, H. (2002). A Principled Approach to Facilitating Distance Education: The Internet, Higher Education and Higher Levels of Learning. Journal of Distance Education, (17) 2, 70-86

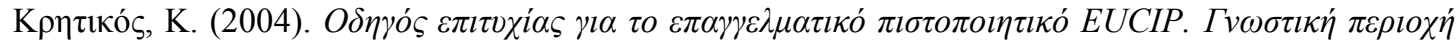

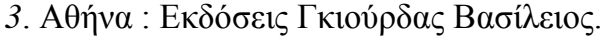

Larreamendy-Joerns, J., \& Leinhardt, G. (2006, winter). Going the distance with online education. Review of Educational Research, 76(4), 567-605.

Lave, J. (1988). Cognition in Practice: Mind, mathematics, and culture in everyday life. Cambridge, UK: Cambridge University Press.

Maki, W., Maki, R., Patterson, M., \& Whittaker, D. (2000). Evaluation of a Web based introductory psychology course: Learning and satisfaction in Web- based versus lecture courses. Behavior Research Methods, Instruments \& Computers, 32, 230-239.

Means, B., Toyama, Y., Murphy, R., Bakia, M. \& Jones, K. (2009). Evaluation of Evidence-Based Practices in Online Learning. A Meta-Analysis and Review of Online Learning Studies. U.S. Department Of Education.

$\Delta \iota \alpha \theta \varepsilon ́ \sigma 1 \mu \mathrm{o} \sigma \tau \mathrm{o}$ http://ifap.ru/library/book440.pdf

Palloff, R. \& Pratt, K. (1999). Building learning communications in cyberspace. San Francisco: Jossey-Bass.

Picciano, A.G. (2002). Beyond student perceptions: Issues interaction, presence, and performance in an online course. Journal of Asynchronous Learning Networks, 6, 20-41.

Pye, J. (1999). Perspectives of ICT in professional development and education.

Information Services \& Use, 19(4), 307-313.

Ryan, S. (2001). Is online learning right for you? American Agent \& Broker, 73(6), 54 - 97

Sapp, D., \& Simon, J. (2005, December). Comparing Grades in Online and Face-to-Face Writing Courses: Interpersonal Accountability and Institutional Commitment. Computers \& Composition, 22(4), 471-489.

Sarason, Y. \& Banbury, C. (2004). Active learning facilitated by using a game-show format or who doesn't. Journal of Management Education, 28 (4), 509-518.

Scriven, M. \& Paul, R. (2004). Defining critical thinking.

$\Delta 1 \alpha \theta \varepsilon \dot{\sigma} \mu_{0} \sigma \tau$ o http://www.criticalthinking.org/aboutCT/definingCT.shtml

Tresman, S. (2002, April). Towards a strategy for improved student retention in programmes of open, distance education: A case study from the Open University UK. The International Review of Research in Open and Distance Learning, 3(1).

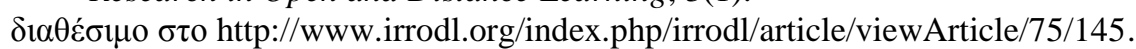

U.S. D. E. (2010). Evaluation of Evidence-Based Practices in Online Learning: A Meta-Analysis and Review of Online Learning Studies.

$\Delta 1 \alpha \theta \varepsilon \dot{\sigma} \mu$ о $\sigma \tau о$

http://www2.ed.gov/rschstat/eval/tech/evidence-based-practices/finalreport.pdf

Vien, C. (2010, January). Connection, Collaboration, and Creation: The Future of Online Learning.

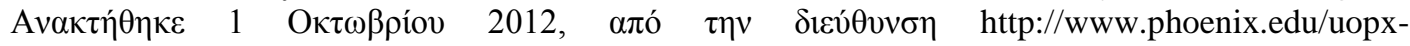
knowledgenetwork/

Vygotsky, L.S. (1978). Mind in Society. Cambridge, MA: Harvard University Press.

Waschull, S. (2005). Predicting Success in Online Psychology Courses: Self-Discipline and Motivation. Teaching of Psychology, 32(3), 190-192.

Watkins, R. (2005). Developing interactive e-learning activities. Performance Improvement, 44, 5-7.

Weller, M. (2000). Reasons for using the web in teaching Proc. 1st Technology in

Teaching and Learning in Higher Education. August 25-27, Samos Island, Greece. 
Wilen-Daugenti, T. (2009). Edu: Technology and learning environments in higher education. New York: Peter Lang.

Woods, R., \& Ebersole, S. (2003). Becoming a "Communal Architect" in the Online Classroom Integrating Cognitive and Affective Learning for Maximum Effect in Web-Based Learning. Online Journal of Distance Learning Administration, 6(1).

$\Delta$ เ $\theta \dot{\varepsilon} \sigma \mu$ o $\sigma \tau \mathrm{h}$ http://www.westga.edu/ distance/ojdla/spring61/woods61.html.

Yates, S. J. (1996). Oral and written linguistic aspects of computer conferencing: A corpus based study. $\Sigma \tau$ o S. C. Herring (Ed.), Computer-mediated communication: Linguistic, social and cross-cultural perspectives (pp. 29-46). Amsterdam: John Benjamins. 